\title{
Modelling and L1 Adaptive Control of Temperature in Biomass Pretreatment
}

\author{
Prunescu, Remus Mihail; Blanke, Mogens; Sin, Gürkan
}

Published in:

Proceedings of 52nd IEEE Conference on Decision and Control

Link to article, DOI:

10.1109/CDC.2013.6760364

Publication date:

2013

Link back to DTU Orbit

Citation (APA):

Prunescu, R. M., Blanke, M., \& Sin, G. (2013). Modelling and L1 Adaptive Control of Temperature in Biomass Pretreatment. In Proceedings of 52nd IEEE Conference on Decision and Control (pp. 3152-3159). IEEE. https://doi.org/10.1109/CDC.2013.6760364

\section{General rights}

Copyright and moral rights for the publications made accessible in the public portal are retained by the authors and/or other copyright owners and it is a condition of accessing publications that users recognise and abide by the legal requirements associated with these rights.

- Users may download and print one copy of any publication from the public portal for the purpose of private study or research.

- You may not further distribute the material or use it for any profit-making activity or commercial gain

- You may freely distribute the URL identifying the publication in the public portal 


\title{
Modelling and $\mathcal{L}_{1}$ Adaptive Control of Temperature in Biomass Pretreatment
}

\author{
Remus Mihail Prunescu${ }^{1}$, Mogens Blanke ${ }^{2}$ and Gürkan Sin $^{3}$
}

\begin{abstract}
Biomass steam pretreatment is a key process in converting agricultural wastes to bioethanol. The pretreatment occurs in a large pressurized tank called a thermal reactor. Two key parameters influence the successfulness of the process: the reactor temperature, and the retention time. A particle pump pressurizes untreated biomass from atmospheric to reactor pressure with recycled steam from the reactor.

This paper formulates a steam mathematical model both for the thermal reactor and the particle pump, which is then used to design an $\mathcal{L}_{1}$ adaptive output feedback controller for the reactor temperature. As steam is recycled from the reactor to pressurize the particle pump, pressure drops and the reactor temperature is disturbed. The main control challenge is to reject these disturbances and keep a steady temperature.

The nonlinear process model embeds mass and energy balances, valve characteristics, and enthalpy-pressure and pressure-temperature dependencies. Nonlinear feed-forward terms are added in the control strategy. The process model, the control strategy, the application of the $\mathcal{L}_{1}$ adaptive controller and its tuning method based on minimizing a cost function represent novelties of this paper.
\end{abstract}

\section{INTRODUCTION}

Biofuel receives more interest lately due to the increase in oil price worldwide, and due to the green commitments that governments have taken for reducing emissions of greenhouse gases, e.g. the Kyoto protocol [1]. In this context, many private companies and governments invest in large scale biofuel production plants [2].

The conversion of agricultural wastes to ethanol requires several steps. A thermal reactor pretreats the biomass by removing the wooden part of the plant, i.e. lignin, and creates a mixture rich in cellulose fibers. Enzymes breakdown the fibers in a liquefaction process and large tanks store the resulting slurry for fermentation. Distillation columns recover ethanol, which is used in preparation of fuel blends. Two more by-products are obtained: lignin, which is recovered from the pretreatment process as bio-pallets and co-combusted in a power plant; and C5 molasses, which is a syrup rich in nutrients and sold to farmers for feeding their animals. The entire biofuel production cycle is thoroughly described in [3], [2].

The biomass pretreatment process is the key step in biofuel production. There are various methods of pretreatment, most

${ }^{1}$ R. M. Prunescu is with the Department of Electrical Engineering, Automation and Control Group, Technical University of Denmark, 2800 Kgs. Lyngby, Denmark rmpr at elektro.dtu.dk

${ }^{2}$ M. Blanke is with the Department of Electrical Engineering, Automation and Control Group, Technical University of Denmark, 2800 Kgs. Lyngby, Denmark mb at elektro.dtu.dk

${ }^{3} \mathrm{G}$. Sin is with the Department of Chemical Engineering, CAPEC, Technical University of Denmark, $2800 \mathrm{Kgs}$. Lyngby, Denmark gs at $\mathrm{kt} \cdot \mathrm{dtu} \cdot \mathrm{dk}$ of them involving steam, strong acids or weak acids [4]. The effects of different pretreatment methods were investigated in [5], [6]. A combination of steam and weak acids gives the best results. Also, to reduce steam operating costs, refineries are integrated with a power plant following the IBUS principle [3].

The steam pretreatment process occurs in a pressurized continuous thermal reactor, which is preceded by a pressurization unit also known as a particle pump [7]. Depending on the load, the particle pump releases an amount of biomass to the thermal reactor with a certain frequency. The degree of pretreatment determines the chemical composition of the outstream. Steam pretreatment started to be investigated in [8] and an empirical pretreatment model for ethanol production was formulated in [9]. Two key parameters of the process, i.e. retention time and reactor temperature, are vital to an effective process.

Various disturbances have been identified for a large thermal reactor in [7], among which the most important one is the particle pump operation cycle, which is causing oscillations in the steam layer temperature. These oscillations further disturb the temperature of the biomass layer, causing an irregular pretreatment process. Temperature matters because a deviation of $10{ }^{\circ} \mathrm{C}$ can cause a drop of $10 \%$ in cellulose recovery [9]. A poor pretreatment can also lead to formation of inhibitors that affect the downstream processes of enzymatic hydrolysis and fermentation, or it could disturb the $\mathrm{pH}$ level of the outstream by releasing more or less acetic acid from the biomass. It is well known that enzymes are sensitive to $\mathrm{pH}$ following a bell-shaped curve [10]. Therefore, the main control challenge in this investigation is to reject any disturbances that affect the reactor temperature in order to ensure a uniform temperature environment in the steam and biomass layers.

To achieve this objective, an adaptive control strategy for the pretreatment temperature is proposed in this article. First, a nonlinear model of the steam layer is formulated from mass and energy balances, enthalpy-pressure and enthalpytemperature dependencies, and nonlinear valve characteristics. Disturbance sources are also introduced, i.e. condensation of steam and uncertainties in the measurement of recycled steam. The biomass load in the reactor is also a factor and the controller will be tuned for a specific load and tested in another operational point. A disturbance can take the system to multiple operational points and adaptation should improve the control performances. Therefore, an adaptive control strategy is developed based on the $\mathcal{L}_{1}$ adaptive output feedback controller. $\mathcal{L}_{1}$ adaptive control represents the latest novelty in control theory [11]. Also, a new tuning method 


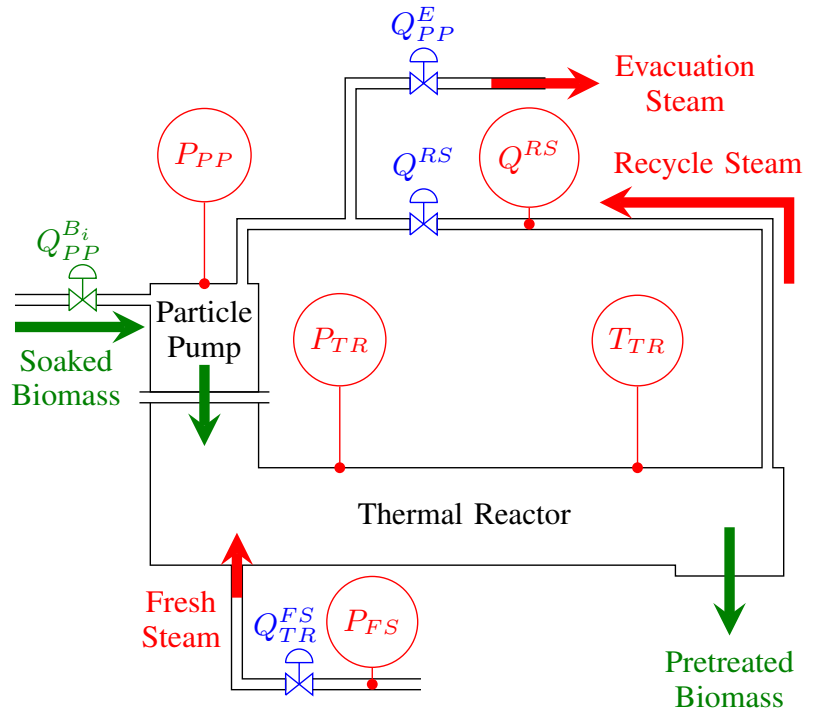

Fig. 1. Process diagram with instrumentation. Green arrows follow the biomass path while red arrows indicate steam flow. There are 3 steam valves, i.e. $Q^{F S}$ or fresh steam valve, $Q^{R S}$ or recycled steam valve and $Q^{E}$ or evacuation valve. $P_{P P}, P_{T R}$ and $P_{F S}$ measure the pressure in the particle pump, thermal reactor and fresh steam pipe, respectively. $T_{T R}$ indicates the reactor temperature.

of the $\mathcal{L}_{1}$ controller is proposed in this paper based on minimizing the integral absolute error (IAE) performance function. The modelling work, the application of the $\mathcal{L}_{1}$ adaptive controller on a biomass pretreatment process and the tuning method of the controller have not been reported earlier in the literature.

\section{PROCESS DESCRIPTION}

The process has been described in [7] and is briefly reiterated in this section. A process diagram is illustrated in Figure 1. The particle pump receives small amounts of soaked biomass and pressurizes them till the reactor pressure. Afterward, the particle pump releases the biomass into the thermal reactor and the cycle repeats. In the thermal reactor, the biomass is pushed horizontally by a snail. The particle pump operates at various frequencies depending on the desired load of the thermal reactor. A fast operation increases the load in the reactor assuming the snail speed constant. When the load increases the available volume for the steam layer diminishes. A reduced air volume can be pressurized faster by steam, so the time constants of the system change according to the load.

It is assumed that the particle pump is pressurized only with recycled steam from the reactor while the thermal reactor is pressurized only with fresh steam, which enters the tank through the bottom. It is preferred to use recycled steam when pressurizing the particle pump because it would create a pressure disturbance that causes a burst of steam from the bottom, which would facilitate the breakdown of soaked biomass leading to a more uniform pretreatment process.

The pressure in the particle pump and the pressure in the reactor are measured as $P_{P P}$ and $P_{T R}$. Another pressure sensor is positioned in the fresh steam pipe and the measurement is denoted as $P_{F S}$. The fresh steam arrives from a flash tank and is assumed to be saturated. The temperature in the thermal reactor is of interest and directly measured as $T_{T R}$. The recycle steam flow is also measured as $Q^{R S}$ and is necessary to construct a feed-forward action. The steam layers from the particle pump and thermal reactor are assumed to be uniform. There are 3 steam valves that can be manipulated and their strokes are denoted as $S_{P P}^{E}, S_{T R}^{F S}$ and $S^{R S}$.

\section{Mathematical Model}

TABLE I

PROCESS MODEL NOMENCLATURE.

\begin{tabular}{|c|c|c|}
\hline \multicolumn{3}{|l|}{ States } \\
\hline$m_{P P}^{S}$ & Steam mass in particle pump (PP) & $\mathrm{kg}$ \\
\hline$h_{P P}^{S}$ & Specific steam enthalpy in PP & $\mathrm{kJ} \mathrm{kg}^{-1}$ \\
\hline$m_{T R}^{S}$ & Steam mass in thermal reactor (TR) & $\mathrm{kg}$ \\
\hline$h_{T R}^{S}$ & Specific steam enthalpy in TR & $\mathrm{kJ} \mathrm{kg}^{-1}$ \\
\hline$m_{P P}^{B}$ & Mass of biomass in PP & $\mathrm{kg}$ \\
\hline$m_{T R}^{B}$ & Mass of biomass in TR & $\mathrm{kg}$ \\
\hline \multicolumn{3}{|l|}{ Inputs } \\
\hline$S_{T R}^{F S}$ & Stroke of fresh steam valve in TR & $\%$ \\
\hline$S^{R S}$ & Stroke of recycle steam valve & $\%$ \\
\hline$S_{P P}^{E}$ & Stroke of evacuation valve in PP & $\%$ \\
\hline$Q_{P P}^{B_{i}}$ & Inflow of biomass in PP & $\mathrm{kg} \mathrm{s}^{-1}$ \\
\hline$Q_{T R}^{B_{i}}$ & Inflow of biomass in TR & $\mathrm{kg} \mathrm{s}^{-1}$ \\
\hline$Q_{T R}^{B_{o}}$ & Outflow of biomass from TR & $\mathrm{kg} \mathrm{s}^{-1}$ \\
\hline$h_{F S}^{S}$ & Fresh steam enthalpy & $\mathrm{kJ} \mathrm{kg}^{-1}$ \\
\hline$P_{F S}$ & Fresh steam pressure & bar \\
\hline \multicolumn{3}{|c|}{ Outputs } \\
\hline$P_{P P}$ & Pressure in PP & bar \\
\hline$P_{T R}$ & Pressure in TR & bar \\
\hline$P_{A}$ & Atmospheric pressure & bar \\
\hline$T_{P P}$ & Temperature in PP & ${ }^{\circ} \mathrm{C}$ \\
\hline$T_{T R}$ & Temperature in TR & ${ }^{\circ} \mathrm{C}$ \\
\hline \multicolumn{3}{|c|}{ Auxiliary Variables } \\
\hline$Q^{R S}$ & Mass flow of recycled steam & $\mathrm{kg} \mathrm{s}^{-1}$ \\
\hline$Q_{P P}^{E}$ & Mass outflow of steam from PP & $\mathrm{kg} \mathrm{s}^{-1}$ \\
\hline$Q_{T R}^{F S}$ & Mass inflow of fresh steam in TR & $\mathrm{kg} \mathrm{s}^{-1}$ \\
\hline$\rho_{P P}^{S}$ & Density of steam in PP & $\mathrm{kg} \mathrm{m}^{-3}$ \\
\hline$\rho_{T R}^{S}$ & Density of steam in TR & $\mathrm{kg} \mathrm{m}^{-3}$ \\
\hline$V_{P P}^{S}$ & Steam volume in $\mathrm{PP}$ & $\mathrm{m}^{3}$ \\
\hline$V_{T R}^{S}$ & Steam volume in TR & $\mathrm{m}^{3}$ \\
\hline \multicolumn{3}{|c|}{ Constant Parameters } \\
\hline$V_{P P}$ & Total volume of PP & $\mathrm{m}^{3}$ \\
\hline$V_{T R}$ & Total volume of TR & $\mathrm{m}^{3}$ \\
\hline \multicolumn{3}{|c|}{ Disturbances } \\
\hline$Q_{T R}^{C}$ & Condensing steam in TR & $\mathrm{kg} \mathrm{s}^{-1}$ \\
\hline$Q_{R S}^{M}$ & Recycle steam measurement noise & $\mathrm{kg} \mathrm{s}^{-1}$ \\
\hline
\end{tabular}

\section{A. Process Model}

The process model consists of mass and energy balances of the steam layer for both the particle pump and the thermal 
reactor:

$$
\begin{aligned}
\frac{d\left(m_{P P}^{S}\right)}{d t} & =Q^{R S}-Q_{P P}^{E} \\
\frac{d\left(m_{P P}^{S} h_{P P}^{S}\right)}{d t} & =Q^{R S} h_{T R}^{S}-Q_{P P}^{E} h_{P P}^{S} \\
\frac{d\left(m_{T R}^{S}\right)}{d t} & =Q_{T R}^{F S}-Q^{R S}-Q_{T R}^{C} \\
\frac{d\left(m_{T R}^{S} h_{T R}^{S}\right)}{d t} & =Q_{T R}^{F S} h_{F S}^{S}-\left(Q^{R S}+Q_{T R}^{C}\right) h_{T R}^{S}
\end{aligned}
$$

Equation (1a) is the particle pump steam mass balance and Equation (1b) is the energy balance of the steam layer from the particle pump. All notations are explained in Table I. Similarly, Equation (1c) shows the mass balance from the thermal reactor and Equation (1d) contains the energy balance of the steam layer in the reactor.

The mass of soaked biomass in both the particle pump and thermal reactor are also tracked as it affects the available volume for steam expansion:

$$
\begin{aligned}
& \frac{d\left(m_{P P}^{B}\right)}{d t}=Q_{P P}^{B_{i}}-Q_{T R}^{B_{i}} \\
& \frac{d\left(m_{T R}^{B}\right)}{d t}=Q_{T R}^{B_{i}}-Q_{T R}^{B_{o}}
\end{aligned}
$$

\section{B. Valve Modelling}

The valve characteristics can be retrieved from the valve manufacturer and shows $K_{V}$ as a function of the valve opening or stroke $S . K_{V}$ represents the flow of steam in $\mathrm{m}^{3} \mathrm{~s}^{-1}$ when the pressure drop across the valve is 1 bar. A typical characteristic is displayed in Figure 2 and can be accurately approximated by a cubic polynomial:

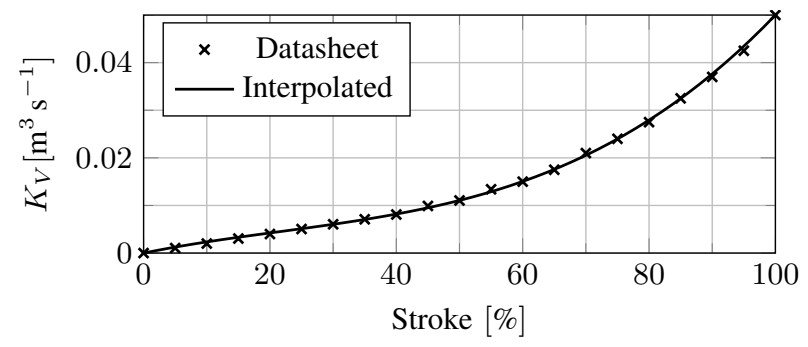

Fig. 2. Steam valve characteristics. Solid line shows the polynomial fit while bullet points indicate measurements taken from the valve datasheet.

$$
K_{V}(S)=k_{3} S^{3}+k_{2} S^{2}+k_{1} S+k_{0}
$$

where $k_{i}$ are the polynomial coefficients and $S$ is the valve stroke.

The fresh steam is, in fact, saturated steam because it is supplied by a flash tank. The steam flow is approximated by:

$$
\begin{aligned}
Q^{S S} & =f\left(S, p_{i}, p_{o}\right)= \\
& = \begin{cases}K_{V}(S) \cdot 22.4 \cdot \sqrt{\left(p_{i}-p_{o}\right) p_{o}} & , p_{o}>\frac{p_{i}}{2} \\
K_{V}(S) \cdot 11.2 \cdot p_{i} & , p_{o}<\frac{p_{i}}{2}\end{cases}
\end{aligned}
$$

where $Q^{S S}$ is the flow of saturated steam, $K_{V}(S)$ is the valve characteristic given in Equation (3), $p_{i}$ is the valve inlet pressure, and $p_{o}$ is the outlet pressure expressed in bar. The recycled steam extracted from the thermal reactor is assumed to be near saturation with a flow approximated by Equation (4).

\section{State Space Model}

State vector $x$ contains:

$$
x=\left[\begin{array}{llllll}
m_{P P}^{S} & h_{P P}^{S} & m_{T R}^{S} & h_{T R}^{S} & m_{P P}^{B} & m_{T R}^{B}
\end{array}\right]^{\mathrm{T}}
$$

Vector $u$ gathers all manipulated variables, i.e. the valve strokes:

$$
u=\left[\begin{array}{lll}
S^{R S} & S_{P P}^{E} & S_{T R}^{F S}
\end{array}\right]^{\mathrm{T}}
$$

The biomass flows are not considered manipulated variables for steam regulation and are placed in vector $\tilde{u}$ :

$$
\tilde{u}=\left[\begin{array}{lll}
Q_{P P}^{B_{i}} & Q_{P P}^{B_{o}} & Q_{T R}^{B_{o}}
\end{array}\right]^{\mathrm{T}}
$$

Disturbances enumerates condensation effects and measurement errors of the recycle steam. Condensation occurs inside the reactor either due to heat losses to the environment or because of temperature differences between the biomass and the steam layer. Measurement errors in the recycle steam flow may appear due to its fast and turbulent dynamics. Vector $d$ comprises all disturbances:

$$
d=\left[\begin{array}{ll}
Q_{T R}^{C} & Q_{R S}^{M}
\end{array}\right]^{\mathrm{T}}
$$

The steam volume in each container is found by subtracting the biomass volume from the total volume:

$$
V_{S}=V-V_{B}
$$

where $V_{S}$ is the steam volume, $V$ is the total volume and $V_{B}$ is the biomass volume. Steam volumes are gathered in vector $v$ :

$$
v=\left[\begin{array}{ll}
V_{P P}^{S} & V_{T R}^{S}
\end{array}\right]^{\mathrm{T}}
$$

Steam densities from both containers are placed into the following vector:

$$
\rho=\left[\begin{array}{ll}
\rho_{P P}^{S} & \rho_{T R}^{S}
\end{array}\right]^{\mathrm{T}}
$$

Steam density is found by dividing the mass of steam by volume. For example, the steam density in the thermal reactor can be computed in the following way:

$$
\rho_{2}=\frac{x_{3}}{v_{2}}
$$

where index 2 and 3 selects the corresponding element from vector $\rho, x$ or $v$.

All pressure variables are gathered in vector $p$ :

$$
p=\left[\begin{array}{llll}
P_{F S} & P_{P P} & P_{T R} & P_{A}
\end{array}\right]^{\mathrm{T}}
$$

where $P_{A}$ is the outlet pressure of the evacuation valve, which is, in fact, atmospheric pressure.

In case of saturated steam, one steam variable is sufficient to determine any of the other variables. In case of wet or superheated steam, 2 steam variables are necessary to determine its state. Steam properties are inferred from the IAPWS IF97 standard. The fresh steam is saturated and 

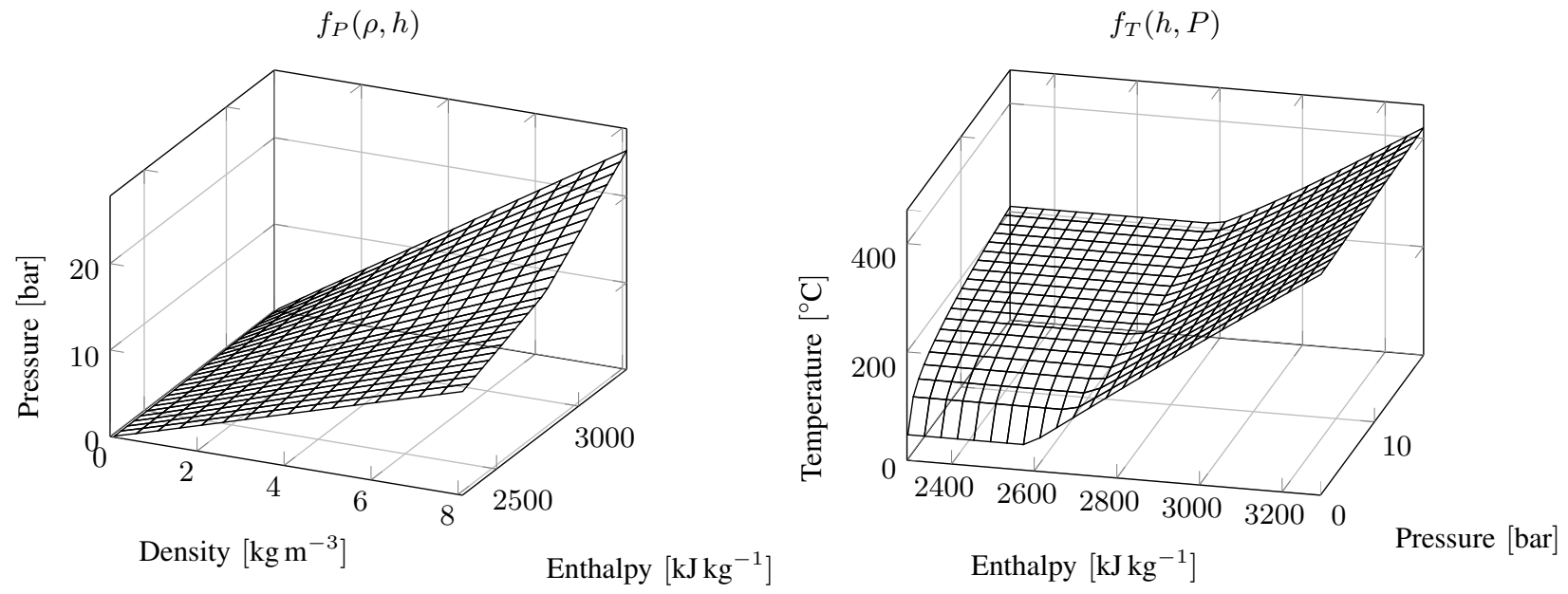

Fig. 3. Steam functions - IAPWS IF97 standard. Left plot shows steam pressure as a function of density and enthalpy while right plot displays steam temperature as a function of enthalpy and pressure.

its enthalpy is found from its pressure, which is directly measured. The reactor pressure is determined from enthalpy and density while the temperature is found from enthalpy and pressure. Figure 3 displays these steam properties. Pressure has a rather linear dependence with respect to density and enthalpy (left plot). Nonlinearities are spotted in temperature variations with respect to pressure in the wet steam region and in the saturation zone (right plot).

Considering the notations introduced above, the state derivatives from (1a), (1c), (1b) and (1d) become:

$$
\begin{aligned}
& \dot{x}_{1}=f\left(u_{1}, p_{3}, p_{2}\right)-f\left(u_{2}, p_{2}, p_{4}\right) \\
& \dot{x}_{2}=\frac{1}{x_{1}}\left[f\left(u_{1}, p_{3}, p_{2}\right)\left(x_{4}-x_{2}\right)\right] \\
& \dot{x}_{3}=f\left(u_{3}, p_{1}, p_{3}\right)-f\left(u_{1}, p_{3}, p_{2}\right)-d_{1} \\
& \dot{x}_{4}=\frac{1}{x_{3}}\left[f\left(u_{3}, p_{1}, p_{3}\right)\left\{g\left(p_{1}\right)-x_{4}\right\}\right] \\
& \dot{x}_{5}=\tilde{u}_{1}-\tilde{u}_{2} \\
& \dot{x}_{6}=\tilde{u}_{2}-\tilde{u}_{3}
\end{aligned}
$$

where $f$ is the function describing the flow through a steam valve shown in Equation (4) and $g$ is the pressure-enthalpy function for saturated steam as found in the IAPWS IF97 standard. States $x_{1-4}$ are coupled with states $x_{5,6}$ through the following equations:

$$
\begin{aligned}
p_{2} & =f_{P}\left(\rho_{1}, x_{2}\right) \\
p_{3} & =f_{P}\left(\rho_{2}, x_{4}\right) \\
\rho_{1} & =\frac{x_{1}}{v_{1}} \\
\rho_{2} & =\frac{x_{3}}{v_{2}} \\
v_{1} & =V_{P P}-x_{5} \rho_{B} \\
v_{2} & =V_{T R}-x_{6} \rho_{B}
\end{aligned}
$$

where $\rho_{B}$ is the soaked biomass density set to $1000 \mathrm{~kg} \mathrm{~m}^{-3}$.

The model outputs are gathered in vector $y$ and comprise the pressure and temperature in both process units:

$$
y=\left[\begin{array}{llll}
p_{P P} & p_{T R} & T_{P P} & T_{T R}
\end{array}\right]^{\mathrm{T}}
$$

where $T_{P P}$ and $T_{T R}$ are calculated as:

$$
\begin{aligned}
& y_{3}=f_{T}\left(x_{2}, p_{2}\right) \\
& y_{4}=f_{T}\left(x_{4}, p_{3}\right)
\end{aligned}
$$

Overall, a nonlinear model with 6 states, 3 inputs and 4 outputs is obtained.

\section{Open Loop Simulation}

An open loop simulation is prepared in order to observe the system response. The simulation scenario is synthesized in Table II.

TABLE II

OPEN LOOP SIMULATION SCENARIO.

\begin{tabular}{rrrr}
\hline Time $[\mathrm{s}]$ & $Q^{F S}[\%]$ & $Q^{R S}[\%]$ & $Q_{P P}^{E}[\%]$ \\
\hline 0 & 0 & 0 & 0 \\
10 & 100 & 0 & 0 \\
30 & 0 & 0 & 0 \\
50 & 0 & 100 & 0 \\
70 & 0 & 0 & 0 \\
90 & 0 & 0 & 100 \\
110 & 0 & 0 & 0 \\
\hline
\end{tabular}

The reactor fresh steam valve is fully opened at time $t=10 \mathrm{~s}$ for $20 \mathrm{~s}$. The recycle steam valve is fully opened at time $t=50 \mathrm{~s}$ for another $20 \mathrm{~s}$. The particle pump evacuation valve is opened at time $t=90 \mathrm{~s}$ for the last $20 \mathrm{~s}$ of the simulation. The results are plotted in Figure 4. Pressurization of the thermal reactor takes a much longer time than the particle pump because it has a larger volume. The reactor pressure resembles a second order system response while the temperature could be approximated by a first order response. The particle pump pressurizes in only several seconds and the pressure in both the reactor and the particle pump equalize. There is a small drop in pressure in the reactor because 
Particle Pump Pressure
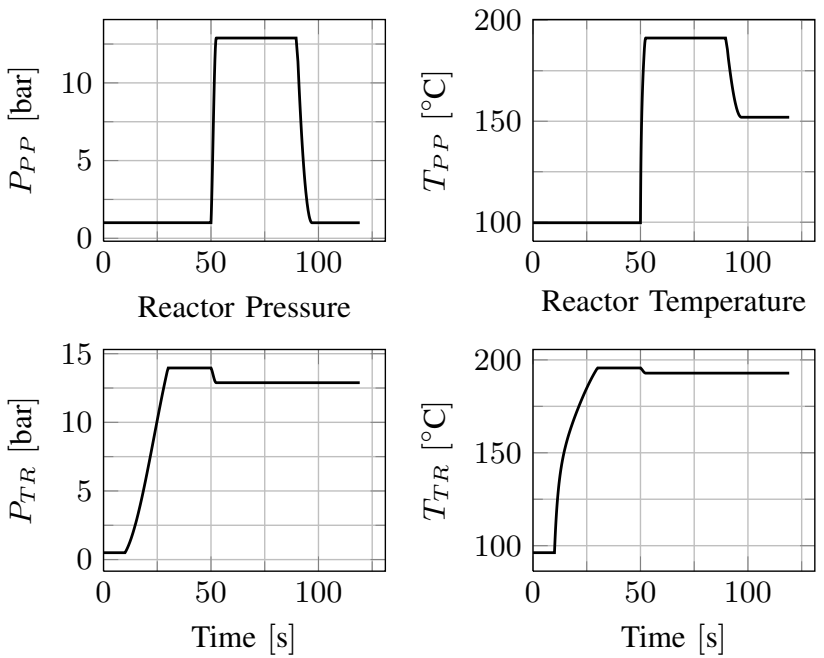

Fig. 4. Open loop simulation. The top plots show the pressure and temperature in the particle pump while the bottom plots display the pressure and temperature in the thermal reactor.

steam is extracted in order to pressurize the particle pump. These disturbances need to be rejected and ensure a steady temperature in the process.

\section{CONTROL DESIGN}

A control strategy is built based on the novel $\mathcal{L}_{1}$ adaptive controller. A nonlinear feed-forward term is also added to the control law based on the measurement or estimation of the recycle steam flow.

Pressurization of the particle pump and its evacuation are achieved by fully opening the recycle and the evacuation valve, respectively. No feedback controllers are required for these actions. The sequence of openings and closings of these valves is determined based on a reference signal $R_{P P}^{P}$ resembling a square wave with a period $\tau_{P P}$. When the reference signal is 1 , the recycle steam valve fully opens and the evacuation valve is closed. When the reference signal is 0 , the recycle valve closes and the evacuation valve opens.

\section{A. Feed-forward Calculation}

The flow of recycled steam can be estimated using the valve model formulated in section III-B or directly measured with a flow sensor. It is desired to find the stroke of the thermal reactor fresh steam valve that would compensate the recycled steam. The stroke of the thermal reactor fresh steam valve is different than the stroke of the recycle valve because the inlet and outlet pressures are different. From Equation (4) the valve characteristic $K_{V}$ can be determined:

$$
K_{V}(t)= \begin{cases}\frac{Q^{R S}(t)}{22.4 \cdot \sqrt{\left(p_{i}-p_{o}\right) p_{o}}} & , p_{o}>\frac{p_{i}}{2} \\ \frac{Q^{R S}(t)}{11.2 \cdot p_{i}} & , p_{o}<\frac{p_{i}}{2}\end{cases}
$$

The valve stroke $S$ can be expressed as a function of the valve characteristic $K_{V}$ by conducting the inverse of function

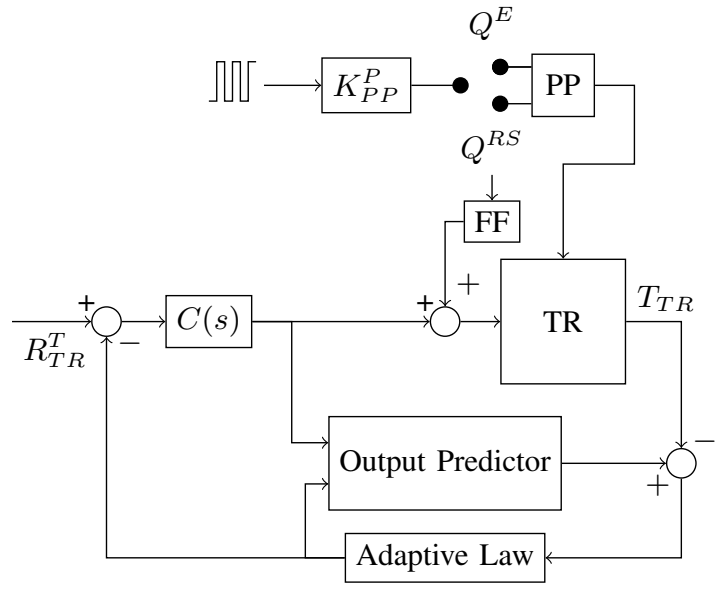

Fig. 5. $\quad \mathcal{L}_{1}$ adaptive output feedback control.

(3). However, for simplification, a linear characteristic is used for this computation:

$$
S_{F F}\left(K_{V}(t)\right)=\frac{100 K_{V}(t)}{0.05}
$$

where 0.05 is the steam volumetric flow (in $\mathrm{m}^{3} \mathrm{~s}^{-1}$ ) when the valve is $100 \%$ open with 1 bar pressure drop.

The stroke $S_{F F}$ is fed-forward through a trust gain $K_{F F}$. The feed-forward control signal becomes:

$$
u_{F F}(t)=K_{F F} S_{F F}(t)
$$

In this application, the trust gain $K_{F F}$ is set to 1 but could be reduced for a more conservative feed-forward action.

\section{B. $\mathcal{L}_{1}$ Adaptive Control}

Figure 5 shows the system in closed loop with an $\mathcal{L}_{1}$ adaptive output feedback controller. The pressurization of the particle pump is shown in the upper part of the figure. There is no feedback action because the pressure in the particle pump should equalize the pressure in the reactor and this objective can be achieved by fully opening the recycle steam valve. The $\mathcal{L}_{1}$ output feedback control strategy is designed for temperature regulation and only one measurement is considered, i.e. $T_{T R}$. The manipulated variable is the fresh steam valve stroke, i.e. $S_{T R}^{F S}$. Therefore, the control object becomes a SISO model.

The $\mathcal{L}_{1}$ output feedback controller consists of an output predictor, an adaptation law and a control filter $C(s)$ [12], [11]. The classical model reference adaptive controller implies a compromise between adaptation and robustness. Moreover, there are no trivial ways of finding a suitable adaptation gain. The $\mathcal{L}_{1}$ adaptive controller separates robustness from fast adaptation by introducing a filter $C(s)$ in the control channel. The analysis of the new controller i.e. the computation of the uniform bounds on outputs and control signals, is performed using the $\mathcal{L}_{1}$ norm, hence the name of $\mathcal{L}_{1}$ adaptive controller.

The open loop process can be expressed as follows [11]:

$$
y_{4}(s)=A(s)\left\{u_{3}(s)+\tilde{d}(s)\right\}
$$




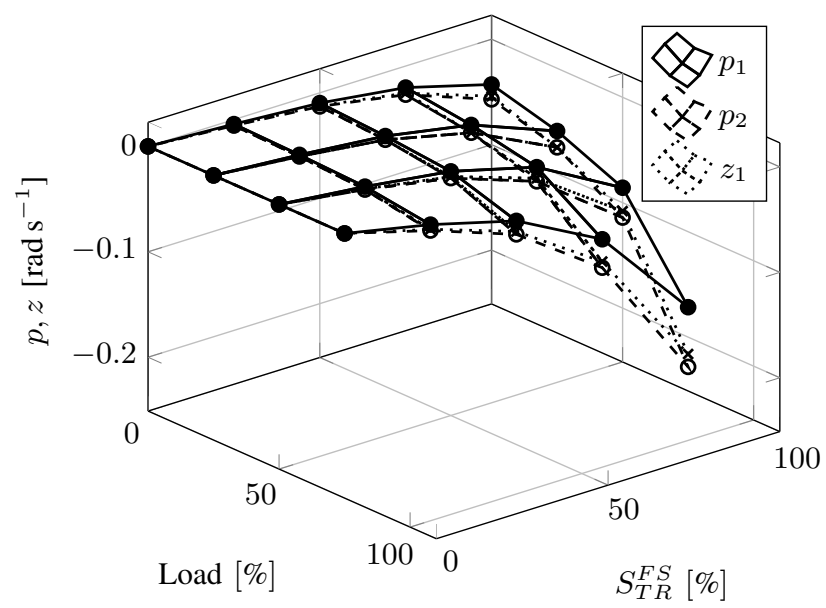

Fig. 6. Poles and zero migration as a function of load and recycle steam valve stroke.

where $y_{4}(s)$ is the reactor temperature, $A(s)$ is an unknown transfer function, $u_{3}(s)$ is the fresh steam valve opening and $\tilde{d}(s)$ lumps all the uncertainties and disturbances that affect $A(s)$. Transfer function $A(s)$ can be approximated as the linearized model around a nominal temperature, e.g. $195^{\circ} \mathrm{C}$, resulting the following general structure:

$$
A(s)=\frac{K\left(s+z_{1}\right)}{\left(s+p_{1}\right)\left(s+p_{2}\right)}
$$

where $K$ is the process gain, $z_{1}$ is a stable zero, and $p_{1}$ and $p_{2}$ are real stable poles. Figure 6 shows the placement of poles $p_{1}$ and $p_{2}$, and zero $z_{1}$ as functions of load and fresh steam valve stroke. The plot was generated for $25 \%, 50 \%$ and $75 \%$ load and for $0 \%, 25 \%, 50 \%, 75 \%$ and $100 \%$ valve strokes. The plot tells that, at low load the zero is canceled by a pole and the resulting system resembles a first order response but as the load and the valve stroke increase, not only the system dynamics become faster as its poles get more negative but also the zero detaches from the pole.

The output predictor contains the desired closed loop dynamics and is chosen as a first order system due to the nature of transfer function $A(s)$ :

$$
\dot{\hat{y}}_{4}(t)=-m \hat{y}_{4}(t)+m\left\{u_{3}(t)+\hat{\sigma}(t)\right\}
$$

where $\hat{y}_{4}(t)$ is the reactor temperature estimation, $m$ is the pole of the closed loop system, $u_{3}(t)$ is the fresh steam valve stroke and $\hat{\sigma}(t)$ is the estimation of all uncertainties and unmodeled dynamics. In frequency domain, the model reference system is denoted as:

$$
M(s)=\frac{m}{s+m}
$$

System (21) can be rewritten in terms of the model reference system [11]:

$$
y_{4}(s)=M(s)\left\{u_{3}(s)+\sigma(s)\right\}
$$

where $\sigma(s)$ is identified as:

$$
\sigma(s)=\frac{\{A(s)-M(s)\} u_{3}(s)+A(s) \tilde{d}(s)}{M(s)}
$$

The idea is to cancel uncertainties $\sigma(s)$ with the help of the control signal $u_{3}(s)$. Therefore, the control signal is defined as [11]:

$$
u_{3}(s)=C(s)\{r(s)-\hat{\sigma}(s)\}
$$

where $C(s)$ is chosen in this application as a first order filter:

$$
C(s)=\frac{c}{s+c}
$$

If Equation (27) is substituted in (25) then:

$$
y_{4}(s)=M(s) C(s) r(s)+M(s)\{\sigma(s)-C(s) \hat{\sigma}(s)\}
$$

If $\sigma(s)$ is perfectly estimated then $\hat{\sigma}(s)=\sigma(s)$ and the disturbances will be rejected only in the bandwidth of $C(s)$.

In [11] the adaptation law is implemented as a projection algorithm. In this application, because there is only one parameter to estimate, the adaptation law is simplified to an integrator:

$$
\dot{\hat{\sigma}}(t)=-\Gamma \tilde{y}_{4}(t)
$$

where $\tilde{y}_{4}(t)=\hat{y}_{4}(t)-y_{4}(t)$ is the estimation error of the output and $\Gamma$ is the integrator gain.

The resulting $\mathcal{L}_{1}$ adaptive output feedback controller has 3 tuning parameters, i.e. the desired closed loop dynamics $m$, the adaptation gain $\Gamma$ and the eigenfrequency of the control filter $c$.

The reference model and the control signal filter can be designed systematically [13]. Assuming perfect knowledge of disturbances, an ideal system $y_{4}(s)$ can be built and used for tuning [13]:

$$
y_{4}(s)=H(s) C(s) r(s)+H(s)\{1-C(s)\} \tilde{d}(s)
$$

where $H(s) C(s)$ is the transfer function from $r(s)$ to $y_{4}(s)$ and $H(s)$ is defined as:

$$
H(s)=\frac{A(s) M(s)}{C(s) A(s)+\{1-C(s)\} M(s)}
$$

Parameters $m$ and $c$ must be chosen such that $H(s)$ is stable and the following $\mathcal{L}_{1}$ norm holds [11]:

$$
\|G(s)\|_{\mathcal{L}_{1}} L<1
$$

where $G(s)=H(s)\{1-C(s)\}$ is the transfer function from $\tilde{d}(s)$ to $y_{4}(s)$ and $L$ is the Lipschitz constant required to guarantee BIBO stability (Lemma 4.1.1 in [11]).

In this application, parameters $m, c$ and $\Gamma$ are found by minimizing the integral absolute error (IAE) function:

$$
\min _{m, c, \Gamma} \int_{0}^{\infty}\left|r(t)-y_{4}(t)\right|
$$

where $r(t)-y_{4}(t)$ is the tracking error and $y_{4}(t)$ is the output of the nonlinear model.

A numerical search procedure is instantiated to obtain parameters $m, c$ and $\Gamma$. The simulation scenario for minimizing the objective function contains the normal operation cycle, i.e. the particle pump extracting steam from the reactor, which causes a disturbance in the reactor temperature. Therefore, the controller is optimized for disturbance rejection. The 
objective function (34) cannot be minimized analytically due to the complexity of the nonlinear model. The numerical search procedure performs various simulations in closed loop, for each simulation evaluating the IAE function from (34). The search algorithm is initialized by following the tuning procedure shown in [14]. A temperature reference of $195^{\circ} \mathrm{C}$ and $0 \%$ biomass load are held in all simulations. The numerical search procedure found the following parameters:

$$
m=0.07 \quad c=20 \quad \Gamma=3135
$$

With these optimal parameters, transfer function $H(s)$ becomes:

$$
H(s)=0.018 \frac{s+20}{(s+5.3)(s+0.07)}
$$

which has stable poles, as required by the design specifications. Transfer function $G(s)$ is:

$$
G(s)=0.018 \frac{s}{(s+0.07)(s+5.3)}
$$

The $\mathcal{L}_{1}$ norm of $G(s)$ is computed and the Lipschitz constant is found:

$$
L=310.64
$$

which ensures BIBO stability of the closed loop system according to Lemma 4.1.1 from [11].

The tracking error between a real and an ideal system, which assumes perfect knowledge of the disturbances, is uniformly bounded with respect to a constant proportional to $1 / \sqrt{\Gamma}[11]$. The larger $\Gamma$ is, the better is the performance. Therefore, a high adaptation gain $\Gamma$ is desired. At the same time, the stability and dynamics of $\hat{\sigma}$ depend on $\Gamma$. The transfer function from $r(s)$ and $d(s)$ to $\hat{\sigma}(s)$ is [13]:

$$
\hat{\sigma}=F(s)[C(s)\{A(s)-M(s)\} r(s)+A(s) d(s)]
$$

where $F(s)$ is identified as:

$$
F(s)=\frac{1}{\frac{s}{\Gamma}+C(s) A(s)+\{1-C(s)\} M(s)}
$$

$\Gamma$ should be chosen such that $F(s)$ is stable. Considering the optimal parameters from (35), the following poles of $F(s)$ are found:

$$
\left[\begin{array}{llll}
-5.04 \pm 9.26 i & -10 & -0.07 & -0.011
\end{array}\right]
$$

which are all stable. Therefore, the adaptation algorithm is stable.

\section{BENCHMARK TESTS}

The $\mathcal{L}_{1}$ adaptive controller is tested in three scenarios that are described below. The feed-forward term is always enabled and the reactor biomass load is set to $25 \%$.

The first scenario corresponds to the normal operation cycle, which assumes pressurization of the particle pump with recycled steam every $180 \mathrm{~s}$. The controller has to keep the reactor temperature at an optimal level, e.g. $195^{\circ} \mathrm{C}$. Condensation of steam cannot be directly measured and is treated as unmeasured disturbance. Steam condensation is modeled as normally distributed white noise with mean $m_{C}$ and standard deviation $\sigma_{C}$ :

$$
Q_{T R}^{C} \in N\left(m_{C}, \sigma_{C}\right)
$$

where $m_{C}=2 \mathrm{~kg} \mathrm{~s}^{-1}$ and $\sigma_{C}=0.3 \mathrm{~kg} \mathrm{~s}^{-1}$.

The second scenario treats measurement noise, which affects the computation of the feed-forward term. The measurement noise specifications are:

$$
Q_{R S}^{M} \in N\left(m_{M}, \sigma_{M}\right)
$$

where $m_{M}=0 \mathrm{~kg} \mathrm{~s}^{-1}$ and $\sigma_{M}=0.5 \mathrm{~kg} \mathrm{~s}^{-1}$.

The temperature reference signal changes during production mode typically when the refinery switches to another type of biomass. Therefore, the temperature controller is also tested with respect to reference step changes in the last simulation scenario.

\section{Results}

The scenario comprising a normal operation with condensation disturbances can be observed in Figure 7. The pressurization of the particle pump is shown in the top left subplot. Two cycles were captured in the plot. The thermal reactor pressure and temperature are displayed in the bottom subplots. Naturally, when the particle pump starts to inhale steam from the thermal reactor, the temperature inside the reactor drops as a consequence of the pressure drop. However, the controller with the feed-forward term is able to reject the disturbance effectively leading to an unnoticeable change in the reactor temperature and pressure as illustrated in the bottom subplots of the figure. The control effort is shown in the top right subplot and is translated into mass flow of fresh steam. It has a non-zero steady-state value due to a non-zero mean value of the condensation disturbance.

In the second simulation scenario, the feed-forward term is considered partially non-reliable and a large noise is injected in the measurement of the recycle steam flow. The results can be observed in Figure 8 . The $\mathcal{L}_{1}$ controller tolerates feed-forward noise effectively. The reactor pressure is not as white as in the first scenario but it translates to negligible variations in the reactor temperature.

The reference tracking case is treated in Figure 9. The feed-forward term has no effect since the particle pump is stopped when changing the reactor temperature. Cooling the reactor is performed by interrupting the supply of fresh steam. The $\mathcal{L}_{1}$ adaptive controller has good performances changing the temperature in less than $1 \mathrm{~min}$, which is satisfactory since temperature set point changes do not occur frequently in production mode.

\section{CONCLUSions}

For the first time, to our knowledge, a steam layer model has been formulated for a particle pump connected to a thermal reactor in biomass pretreatment. The process model embeds nonlinear valve characteristics and steam states dependencies, e.g. pressure, temperature, enthalpy and density.

The developed control strategy based on the novel $\mathcal{L}_{1}$ adaptive output feedback controller proved to have very 
Particle Pump Pressure

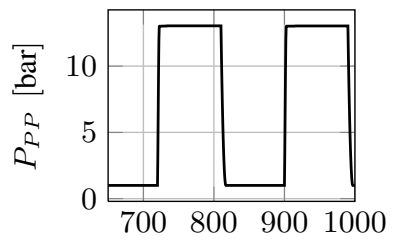

Reactor Pressure

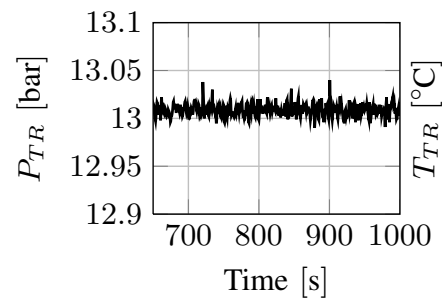

Fig. 7. Normal operation cycle with condensation noise.

Particle Pump Pressure
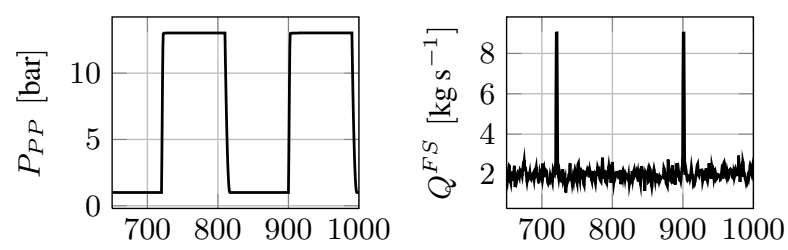

Reactor Pressure

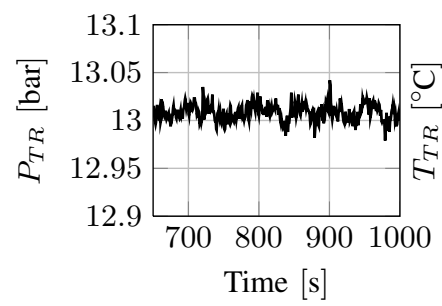

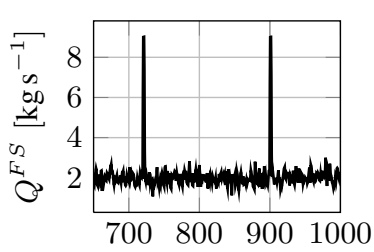

Reactor Temperature

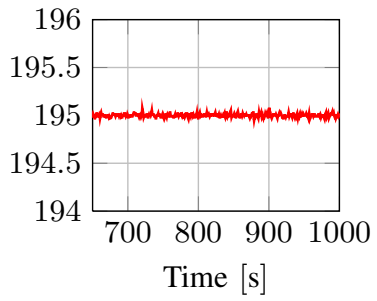

good performances in all simulation scenarios. Measurement noise that affects the feed-forward term and condensation disturbances were handled satisfactory. The application of such a controller to regulate the temperature in a biomass pretreatment reactor has not been reported earlier in the literature.

The last achievement of the paper is the tuning method of the $\mathcal{L}_{1}$ adaptive controller. It proved successfully to formulate the tuning of the controller as an optimization problem. The integral absolute error (IAE) has been used as an objective function and the determined controller parameters proved to give satisfactory results in simulation.

The temperature controller developed in this paper is part of an inner control loop layer of a biorefinery. The temperature setpoint will be given by an outer control loop layer that is using the pretreated biomass composition as feedback. The objective is to obtain a steady pretreatment process and any variations in biomass composition or type can be rejected by changing the temperature in the reactor.

\section{REFERENCES}

[1] Kyoto Protocol to the United Nations Framework Convention on Climate Change, United Nations Std.

[2] J. Larsen, M. Ø. Petersen, and L. Thirup, "Inbicon makes lignocellulosic ethanol a commercial reality," Biomass and Bioenergy, vol. 46, pp. $36-45,2012$.

[3] J. Larsen, M. Ø. Petersen, L. Thirup, H. W. Li, and F. K. Iversen, "The ibus process - lignocellulosic bioethanol close to a commercial reality," Chemical Engineering \& Technology, vol. 5, pp. 765-772, 2008.

[4] S. Zabihi, R. Alinia, F. Esmaeilzadeh, and J. F. Kalajahi, "Pretreatment of wheat straw using steam, steam/acetic acid and steam/ethanol and its enzymatic hydrolysis for sugar production," Biosystems Engineering, vol. 105, pp. 288-297, 2010.

[5] N. Mosier, C. Wyman, B. Dale, R. Elander, Y. Lee, M. Holtzapple, and M. Ladisch, "Features of promising technologies for pretreatment of lignocellulosic biomass," Bioresource Technology, vol. 96, pp. 673-686, 2005.

[6] D. Chiaramonti, M. Prussi, S. Ferrero, L. Oriani, P. Ottonello, P. Torre, and F. Cherchi, "Review of pretreatment processes for lignocellulosic ethanol production, and development of an innovative method," Biomass and Bioenergy, vol. 46, pp. 25-35, 2012.

Fig. 8. Normal operation cycle with measurement noise.

Particle Pump Pressure

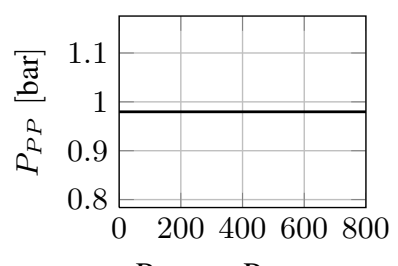

Reactor Pressure

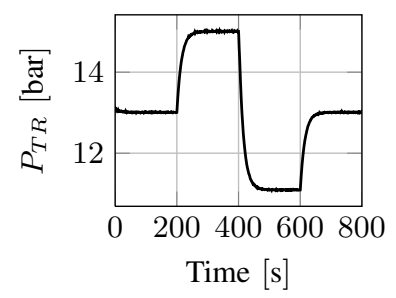

Fresh Steam Inflow
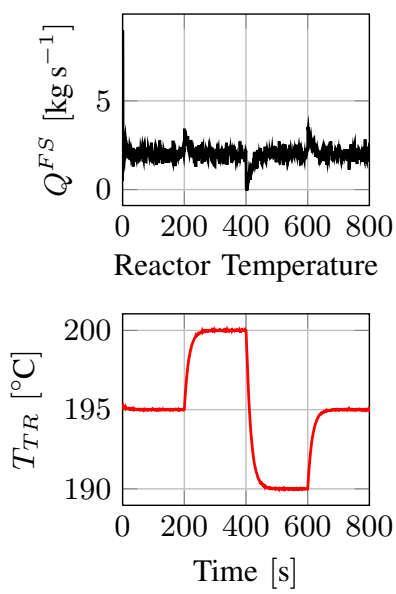

[7] R. M. Prunescu, M. Blanke, J. M. Jensen, and G. Sin, "Temperature modelling of the biomass pretreatment process," Proceedings of the 17th Nordic Process Control Workshop, pp. 8-17, 2012.

[8] R. P. Overend, E. Chornet, and J. A. Gascoigne, "Fractionation of lignocellulosics by steam-aqueous pretreatments," Philosophical Transactions of the Royal Society of London. Series A, Mathematical and Physical Sciences, vol. 321, No. 1561, pp. 523-536, 1987.

[9] М. Ø. Petersen, J. Larsen, and M. H. Thomsen, "Optimization of hydrothermal pretreatment of wheat straw for production of bioethanol at low water consumption without addition of chemicals," Biomass and Bioenergy, vol. 33, pp. 834-840, 2009.

[10] P. D. Fullbrook, "Practical limits and prospects (kinetics)," Industrial Enzymology 2nd Edition, pp. 505-540, 1996.

[11] N. Hovakimyan and C. Cao, $\mathcal{L}_{1}$ Adaptive Control Theory - Guaranteed Robustness with Fast Adaptation. SIAM, 2010.

[12] C. Cao and N. Hovakimyan, " $\mathcal{L}_{1}$ adaptive output feedback controller for systems of unknown dimensions," IEEE Transactions on Automatic Control, vol. 53, pp. 815-821, 2008.

[13] R. Hindman, C. Cao, and N. Hovakimyan, "Designing a high performance, stable $\mathcal{L}_{1}$ adaptive output feedback controller," AIAA Guidance, Navigation and Control Conference and Exhibit, vol. 1, p. 6644, 2007.

[14] R. M. Prunescu, M. Blanke, and G. Sin, "Modelling and $\mathcal{L}_{1}$ adaptive control of ph in bioethanol enzymatic process," Proceedings of the American Control Conference 2013, pp. 1888-1895, 2013.

Fig. 9. Reference tracking with condensation noise. 